\title{
Peripheral Cemento-Ossifying Fibroma
}

\author{
Dr S. Anitha Devi, Dr K.Sasi Rekha, Dr Venkata Srikanth, Dr M J. Renganath, \\ Dr R. Dhivya
}

\begin{abstract}
Cemento-ossifying fibroma is a benign, fibro-osseous tumor belonging to the same category as fibrous dysplasia and cemento-osseous dysplasia. It is a reactive gingival overgrowth occurring frequently in the mandibular premolar-molar region. A patient aged 15 years old with the chief complaint of a swelling in relation to 12 and 13 region is presented in this paper. The lesion was asymptomatic, pinkish and pedunculated. Histologically, it showed a cellular, fibrous connective tissue stroma with calcifications. The lesion was histologically similar to peripheral ossifying fibroma and complete surgical removal of the lesion was carried out under local anesthesia
\end{abstract}

\section{Introduction}

Gingival growths are one of the most frequently encountered lesions in the oral cavity. Most of these lesions, such as irritational fibroma, pyogenic granuloma, peripheral ossifying fibroma and peripheral giant cell granuloma, are innocuous and rarely present with aggressive features ${ }^{[1]}$

Ossifying fibroma is a benign neoplasm arising in craniofacial bones, composed of proliferating fibroblasts with osseous products that include bone and ovoid calcifications; these lesions are well demarcated from the adjacent bone. There are two types of ossifying fibromas: the central type and the peripheral type. The central type arises from the endosteum or the periodontal ligament adjacent to the root apex and causes expansion of medullary cavity. The peripheral type occurs solely on the soft tissues covering the tooth-bearing areas of the jaws ${ }^{[2]}$

In 1872, Menzel first described ossifying fibroma; but only in 1927, Montgomery assigned a terminology to it ${ }^{[2]}$. It is widely considered that the lesion often associated with trauma or local irritants such as sub gingival plaque and calculus, dental appliances and poor dental restorations ${ }^{[3]}$. A slight female predominance has been seen in a literature review by Daley ${ }^{[4]}$

The pathogenesis of this tumor is uncertain. Due to their clinical and histopathological similarities, some peripheral cemento-ossifying fibromas are believed to develop initially as a pyogenic granuloma that undergoes fibrous maturation and subsequent calcification ${ }^{[2] .}$

Ossifying fibroid epulis; peripheral fibroma with calcification; peripheral cemento-ossifying fibroma; and calcifying fibroma are the terms which refer to peripheral ossifying fibroma (POF). Peripheral cementoossifying fibroma appears as a nodular mass, either pedunculated or sessile. It most commonly appears to originate from interdental papilla. The color ranges from red to pink, and the surface is frequently but not always ulcerated. There is a slight predilection for the maxillary arch (60\%) and the incisor cuspid region $(50 \%)$. The treatment of choice is surgical excision with removal of irritation factors.

\section{Case report}

A 15 year old male patient reported with the complaint of pain and swelling in upper right front tooth region for past 4 month. Which started as a small papule approximately 4 months ago and gradually increased in size with time to attain present size and with no medical and dental history.

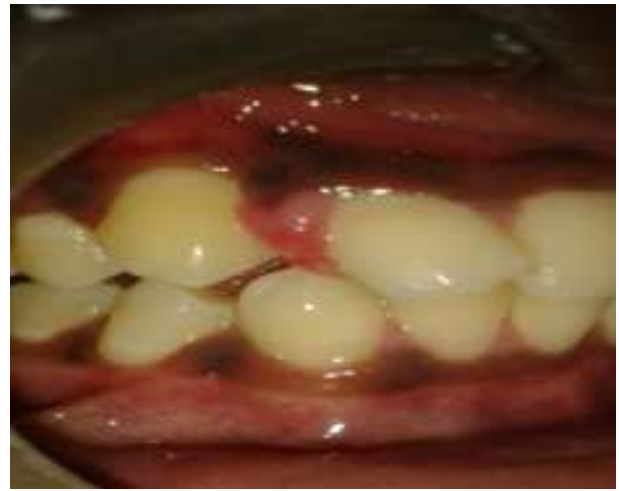

Fig.1: Clinical presentation of the lesion, labial view in relation to 12 and 13 . 
Intraoral examination revealed an approximately $1.5 \mathrm{~cm} \times 1.5 \mathrm{~cm}$ sessile, non-tender, firm, pale pinkish growth present on the interdental gingiva in relation 12 and 13 region[Fig.1 and 2]

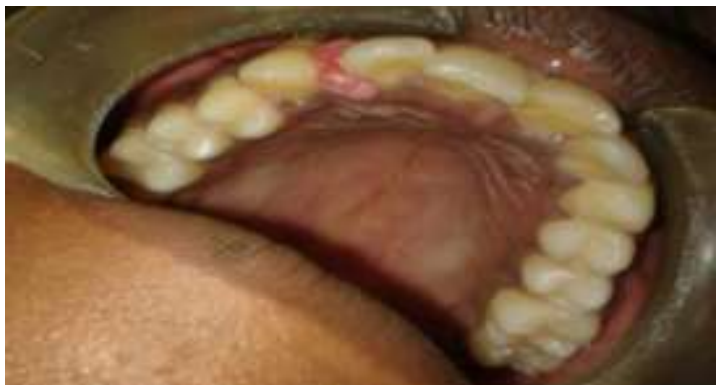

Fig.2: Clinical presentation of the lesion, palatal view in relation to 12 and 13 .

Radiographically, there was horizontal bone loss in relation to maxillary right front tooth region [Fig.3]

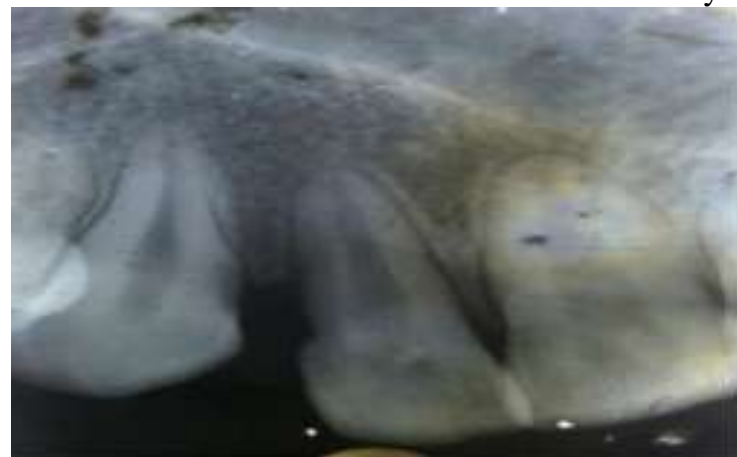

Fig.3: Intraoral periapical radiograph of 12 and 13 showing bone loss

The provisional diagnosis of irritation fibroma was made. The periodontal treatment plan included patient education and motivation for oral hygiene instructions, scaling and root planing, reevaluation for surgical excision of the lesion under anesthesia. Scaling and root planing was performed for elimination of local etiological factors. After 1 week of scaling and root planing, re-evaluation and surgical excision was performed [Fig.4and5] and periodontal dressing was placed with suture [Fig. 6 and 7]

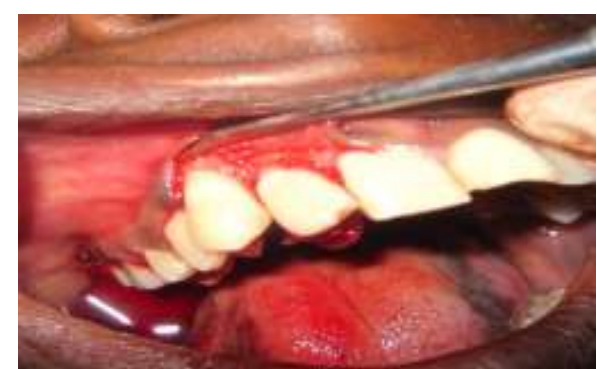

Fig.4: During excision - labial view

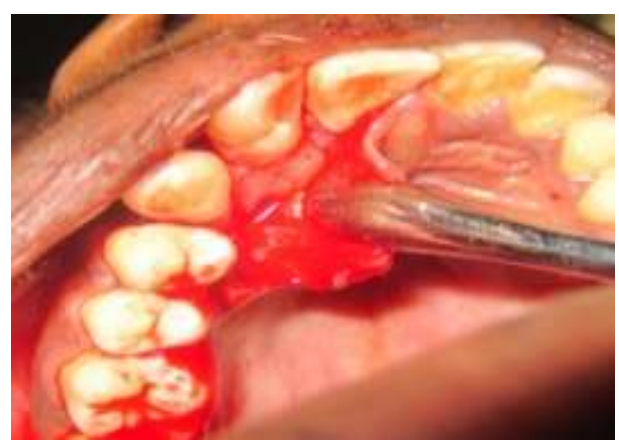

Fig.5: During excision palatal view 


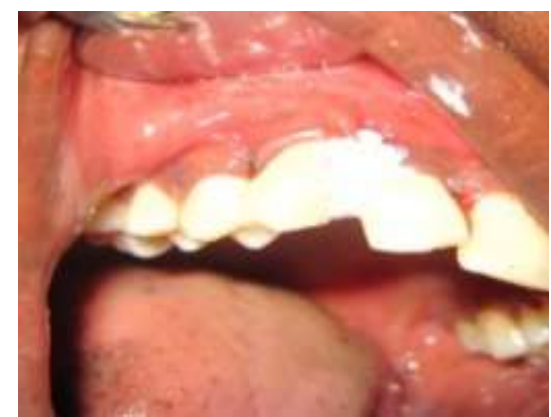

Fig.6: Periodontal dressing placed in labial view

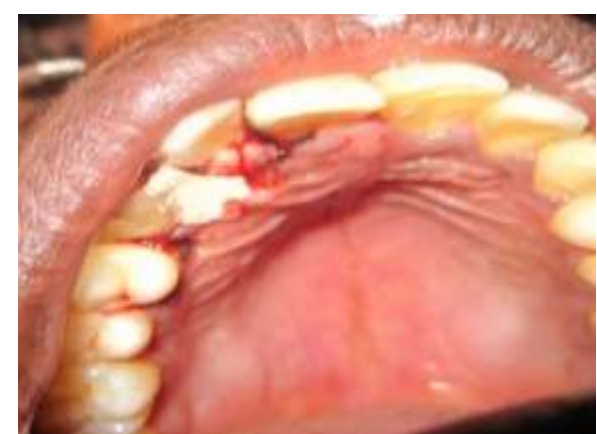

Fig.7: Periodontal dressing placed in palatal view

Patient was given post-operative instructions and was prescribed with analgesic (Tab. Combiflam for 3 days and antimicrobial rinse $(0.2 \%$ Chlorhexidine gluconate twice a day for 1 week).

Patient was recalled, after 1 week for follow-up and one month follow up[Fig.12 and13]

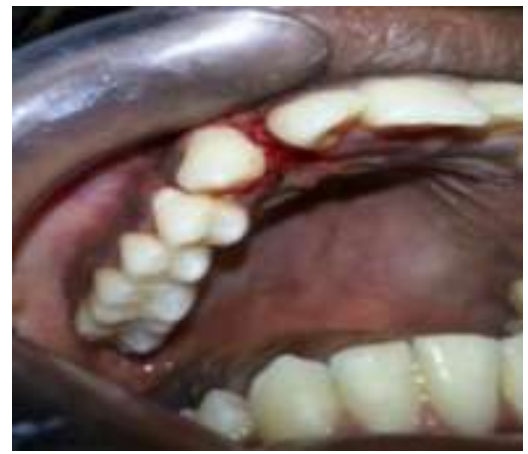

Fig.8: Post-operative 1 week showing uneventful recovery

The excised tissue was placed in $10 \%$ formalin and sent for the histopathologic examination. [Fig.9]

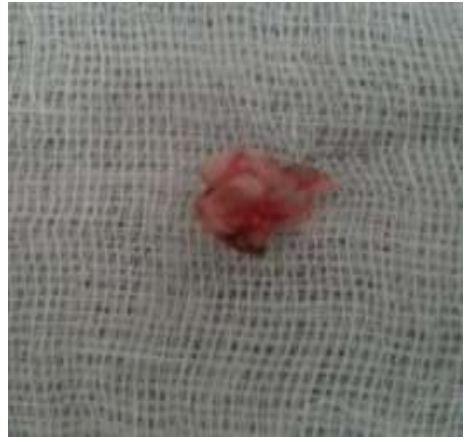

Fig.9: Excised lesion

Biopsy specimen microscopically The given $\mathrm{H}$ and $\mathrm{E}$ stained soft tissue section shows a fibrous proliferation associated with stratified squamous parakeratinised epithelium. The epithelial retepegs appear short. The connective tissue shows dense fibroblastic proliferation and vascular proliferation. The blood vessels are filled with RBC's. Mineralised masses are found to be scattered in the entire connective tissue. Trabecular 
and woven bone like material are seen along with droplets of basophilic cementum like material. Few areas also show irregular mass of basophilic material suggestive of dysplastic calcification [Fig10and11]

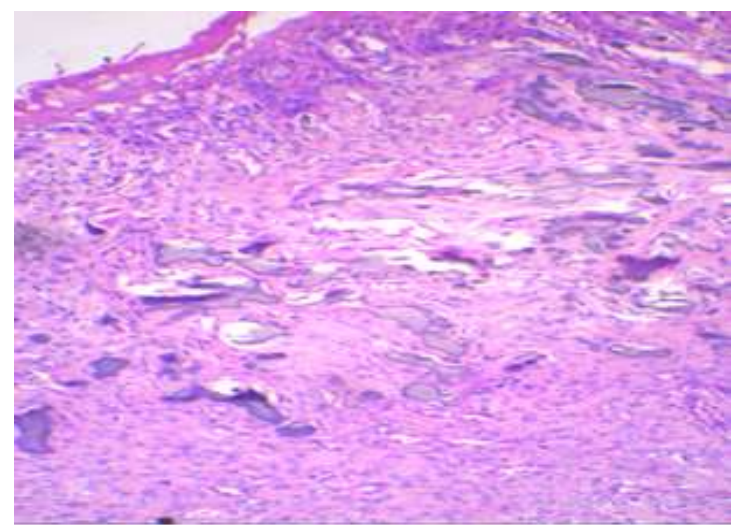

Fig.10: Histopathological slide showing basophilic cementum-like material

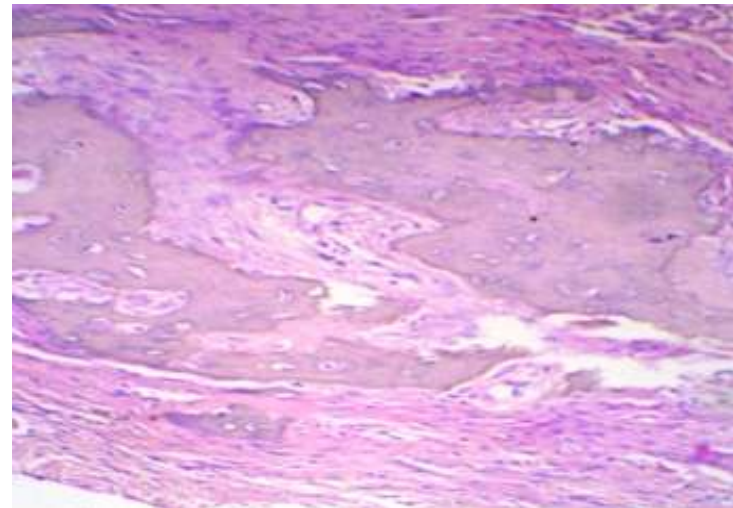

Fig.11: Histopathological slide showing woven bone

\section{Discussion}

Ossifying fibroma was first described by Menzel in 1872. It is a rare, benign primary bone tumour that occurs most commonly in the jaw. Montgomery in1927 coined the term "ossifying fibroma" ${ }^{[5]}$ The peak incidence is found most frequently in teenagers, young adults and women are 2-4 times more likely to be affected than men ${ }^{[6]}$

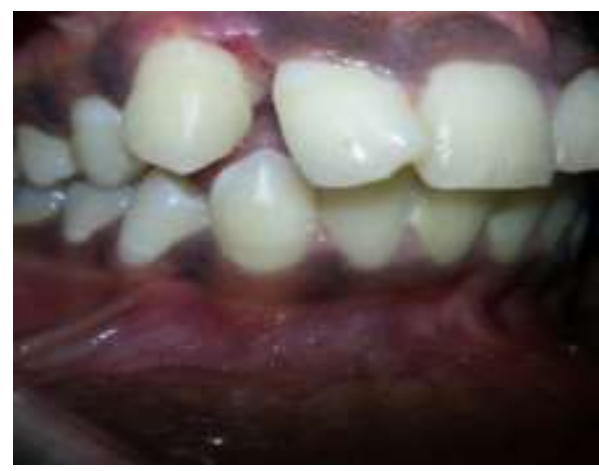

Fig.12: Post-operative 3 months in labial view 


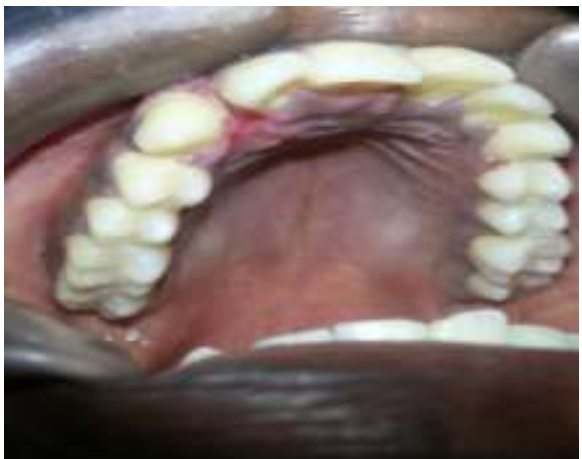

Fig.13: Post-operative 3 months in palatal view

There are two types of ossifying fibroma, central and the peripheral. But POF is not a counterpart of the central ossifying fibroma but reactive lesion of the gingiva. It usually presents as relatively uncommon, solitary, non neoplastic gingival growth. Clinically the lesion may be sessile or pedunculated with smooth, ulcerated or erythematous surface. Various terminologies have been used in the literature to describe this lesion like peripheral fibroma with calcifications, peripheral odontogenic fibroma, ossifying fibro-epithelial polyp, peripheral fibroma with osteogenesis, peripheral fibroma with cementogenesis, calcifying fibroblastic granuloma and calcifying or ossifying fibrous epulis ${ }^{[7]}$

Radiologically, these tumors may present a number of patterns depending on their degree of mineralization. Two basic patterns have been defined: one characterized by the presence of a unilocular or multilocular radio transparent image, and another showing mixed density due to a variable internal amount of radiopaque material. The margins of the lesion are relatively well defined and present a peripheral osteocondensation zone. The fibroma is of concentric appearance within the medullary zone of the bone, and the cortical layers are preserved. In some cases the lesions are seen to be associated to root reabsorption and displacement of the roots of the neighbouring teeth. ${ }^{[8]}$

Histologically, the POF appears to be a nonencapsulated mass of cellular fibroblastic connective tissue of mesenchymal origin, covered with stratified squamous epithelium, which is ulcerated in $23 \%-66 \%$ of cases. Most ulcerated lesions

occur in patients in the second decade. POFs contain areas of fibrous connective tissue, endothelial proliferation and mineralization. Endothelial proliferation can be profuse in the areas of ulceration, which can be misleading in clinical diagnosis, as the lesion may appear to be a pyogenic granuloma. The mineralized component of POF varies ${ }^{[9]}$ These finding are in accordance with our case report.

\section{Conclusion}

POF is a slowly progressing lesion, the growth of which is generally limited. Many cases will progress for long periods before patients seek treatment because of the lack of symptoms associated with the lesion. The prognosis is usually good, since recurrences are not frequent. This was confirmed in our case, since repair of treated surgical site was seen to be asymptomatic in 3 month follow-up, though longer follow-up is required.

\section{Reference}

[1]. Pal S, Hegde S, Ajila V. The varying clinical presentations of peripheral ossifying fibroma: A report of three cases. Rev Odonto Cienc 2012;27(3):251-55.

[2]. Chatterjee A, Ajmera N, Singh A. Peripheral cemento-ossifying fibroma of maxilla.Journal of Indian Society of Periodontology 2010; 14(3):186-89

[3]. Pendyala G, Joshi S, Marawar P P, Pawar B, Mani A.Peripheral ossifying fibroma . Pavara med Rev 2012;4(2):27-30.

[4]. Baiju CS, Rohatgi. Peripheral odontogenic fibroma: A case report and review. Journal of Indian Society of Periodontology 2011;159(3):273-75.

[5]. Agrawal S M, barodiya A, agrawal M G. Ossifying fibroma of mandible: a case report. Njdsr 2012;1:11-13.

[6]. Jamin J P, maheshwari T N U. An unusual clinical presentation of multifocal peripheral cemento-ossifying fibroma - a case report. Journal of oral medicine, oral surgery, oral pathology and oral radiology 2015;1(2):99-102.

[7]. Sunil P R D, raj AP N, kumar S, darshan S V, raju M V. A case report of peripheral cemento-ossifying fibroma in maxillary anterior palatal region. URJDS 2014;02 (01): 3-5.

[8]. Ghani B, Bhattacharya H S, manjunath R G S, khan R. peripheral cemento-ossifying fibroma -a case report. National Journal of Medical and Allied Sciences $2014 ; 3$ (1):42-45.

[9]. Farquhar T MacLellan J, Dyment H, Anderson R S. Peripheral Ossifying Fibroma: A Case Report. JADC 2008;74:809-12. 